\title{
Risk of Lymphoma Associated with Anti-TNF Therapy in Patients with Inflammatory Bowel Disease: Implications for Therapy
}

This article was published in the following Dove Press journal: Clinical and Experimental Gastroenterology

\section{Jessica Dahmus \\ Michelle Rosario \\ Kofi Clarke (ID}

Gastroenterology, Penn State Milton S. Hershey Medical Center, Hershey, PA, USA

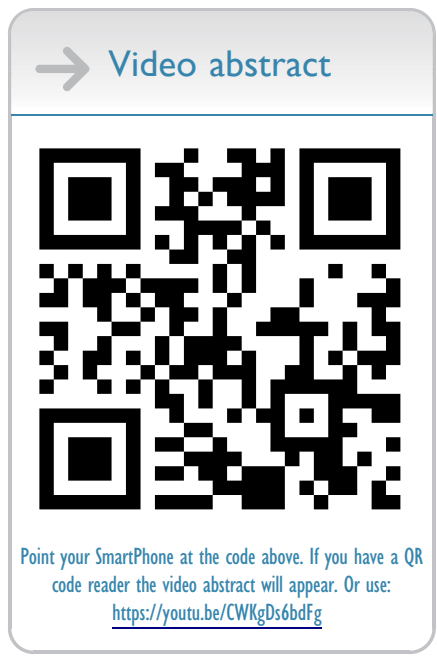

Correspondence: Kofi Clarke

Tel +| 7|7-53|-874|

Fax + I 7|7-53I-6770

Email kclarke@pennstatehealth.psu.edu

\begin{abstract}
Anti-tumor necrosis factor alpha (ATA) therapy plays a significant role in the treatment of moderate to severe inflammatory bowel disease (IBD). There are concerns regarding risks associated with their use, including malignancy and, specifically, lymphoma. Many previous studies have sought to determine whether there is a true link between ATA therapy in IBD and development of lymphoma. However they have been hindered by short follow-up times, few cases, and confounding factors such as previous thiopurine exposure. This review seeks to update the literature by evaluating more recent studies assessing the link between ATA monotherapy and lymphoma development. It also summarizes findings of those studies and provides additional clinical guidance pertaining to this class of biologic therapy.
\end{abstract}

Keywords: malignancy, cancer, biologics, ulcerative colitis, Crohn's disease, hepatosplenic T-cell lymphoma

\section{Introduction}

Since the introduction of infliximab (IFX) in 1998 for use in the treatment of Crohn's disease (CD), and subsequent approval for use in ulcerative colitis (UC) in 2006, anti-tumor necrosis factor-alpha inhibitor (ATA) therapy has become a key component of the treatment armamentarium for moderate to severe inflammatory bowel disease (IBD). However, there are concerns regarding potential complications related to ATA therapy, including malignancy. Cancers associated with ATA include solid organ malignancies, nonmelanoma skin cancers, melanoma, lymphoproliferative malignancies, and viral-associated lymphomas, such as Epstein-Barr Virus (EBV). Proposed mechanisms of tumorigenesis with ATA drugs include decreased activation of natural killer (NK) cells, promotion of apoptosis among macrophages and T cells, induction of cell cycle arrest of T cells, and inhibition of IL-1 $\beta$ production by monocytes. ${ }^{1-4}$

Historically, one of the first observational studies to note a potential link between ATA use and malignancy risk was in 2002. It described 26 cases of lymphoproliferative disease in a patient cohort receiving etanercept (18 cases) or IFX (8 cases) for either rheumatoid arthritis (RA) or IBD (5 cases). ${ }^{5}$ This association was appreciated about two and a half years after the drugs were licensed for clinical use in the United States. In that study, $81 \%$ of the 26 cases were non-Hodgkin's lymphomas (NHL). Interestingly, most of the cases were detected within 8 weeks of initiation of ATA 
therapy. Three of the 26 patients had a history of lymphoproliferative disease prior to initiation of the ATA. Seventythree percent of subjects had previous or concurrent immunomodulator medication (IMM) exposure, including methotrexate, azathioprine, and 6-mercaptopurine.

Multiple additional analyses have been published addressing the concern over a link between ATA therapy and lymphoma with inconsistent findings. However, many of the data supporting a link between ATA use and lymphoma development may have been confounded by a patient's previous thiopurine (TP) exposure. Additionally, results may have been extrapolated from the rheumatoid arthritis literature which is a disease known for its inherent risk of B-cell lymphoma development. ${ }^{6}$ Some studies were limited by short term follow-up or underpowered sample sizes, leading to dissimilar findings amongst studies. ${ }^{7}$ Despite lack of discrete conclusions, the FDA has maintained a black box warning concerning lymphoma risk with ATA therapy.

The American Gastroenterological Association (AGA) published its

Technical Review on the Use of Thiopurines, Methotrexate, and Anti-TNF- $\alpha$ Biologic Drugs for the Induction and Maintenance of Remission in Inflammatory Crohn's Disease

in 2013. ${ }^{8}$ It included an evaluation of the current literature surrounding the risk of lymphoma in $\mathrm{CD}$ populations exposed to TP, methotrexate, ATA monotherapy, and ATA/TP combination therapy. Much of the analyzed data relating to lymphoma risk was from the TREATTM Registry, CESAME, and Kaiser studies. ${ }^{9-11}$ These aforementioned studies, in sum, appeared to indicate a small but appreciable association between TP or combination therapy use and lymphoma development. The recommendations did not appreciate an increased risk of lymphoma with ATA monotherapy, although this was deemed to be of very low-quality evidence due to bias, imprecision, and limited follow-up data. With this in mind, the AGA recommended further studies to assess the risk of lymphoma relative to ATA monotherapy.

Since the first observational study among RA and IBD patients in 2002, the concern of an increased risk of lymphoma development with ATA monotherapy has persisted and has undergone further investigations. ${ }^{5}$ This qualitative review aims to evaluate and analyze the relevant literature addressing this quandary since publication of the AGA guidelines in 2013. Although this time span may seem arbitrary, it was chosen to allow a closer look at more recently published literature, which benefitted from longer follow-up times, larger sample sizes, and potentially fewer subjects with previous exposure to TP therapy, allowing for a more clear evaluation of ATA monotherapy and lymphoma development.

Although the main focus of this review is to assess ATA monotherapy exposure and the risk of lymphoma development in studies since 2013, additional focus will be given to addressing additional issues with IBD management in the biologic era such as the risk of hepatosplenic T-cell lymphoma (HSTCL), ATA use in patients with current or previous cancer, and implications for therapy in the discussion section.

\section{Materials and Methods}

\section{Literature Search and Study Selection}

A literature search was performed utilizing PubMed, Cochrane review, and OVID Medline. Two authors assessed articles from January 1, 2013 to April 28, 2020. A combination of search terms were used, including "malignancy," “cancer," "lymphoma," "inflammatory bowel disease," "Crohn's disease," "ulcerative colitis," "anti-tumor necrosis factor," "anti-TNF," "certolizumab", "infliximab", "adalimumab", and "golimumab". A specific focus was then placed on assessing articles that addressed the risk of lymphoma associated with ATA inhibitor monotherapy use in inflammatory bowel disease patients.

Selected studies included randomized control trials, observational studies, systematic reviews, and metaanalyses that met timeline, search term, review focus, and exclusion criteria. Studies on non-human subjects, pediatric patients, non-English written articles, and those excluding IBD patients were not included in our qualitative review. Publications that did not address lymphoma risk of ATA therapy were not included in the main results but were mentioned in the discussion section for background and added informational purposes.

\section{Results}

The search strategy yielded over 1000 citations. After excluding duplicate articles, and articles that did not meet the inclusion criteria, 16 articles remained and were analyzed in detail. These included two meta-analyses, four retrospective cohort studies, four prospective cohort studies, three nested casecontrol studies, two cross-sectional studies, and one post hoc analysis. Table 1 summarizes the articles' characteristics and findings. Immunomodulators (IMM) included medications 


\begin{tabular}{|c|c|c|c|c|c|c|c|}
\hline 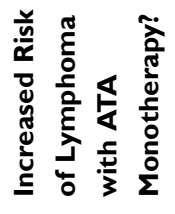 & 우 & z & 우 & zo & $\stackrel{0}{z}$ & ż & z \\
\hline 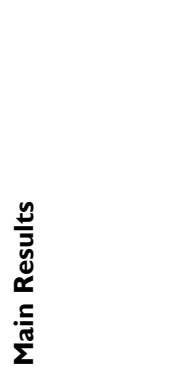 & 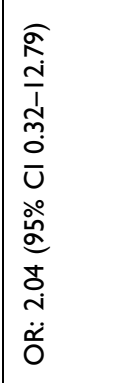 & 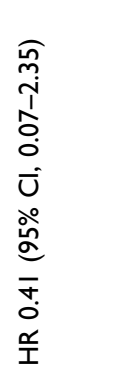 & 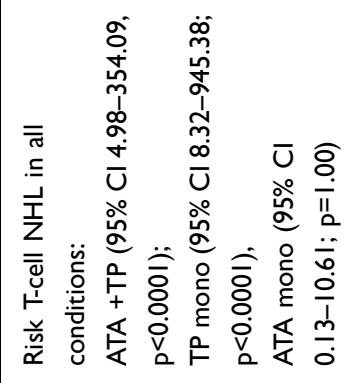 & 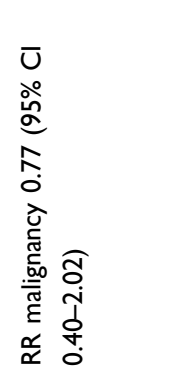 & 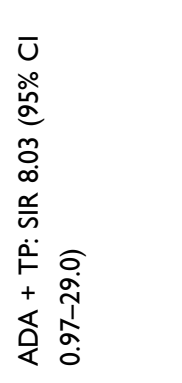 & 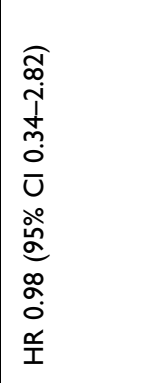 & 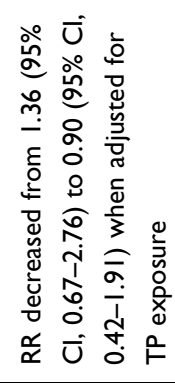 \\
\hline 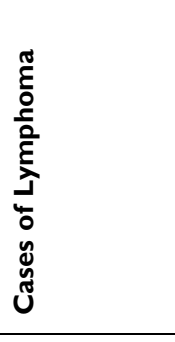 & 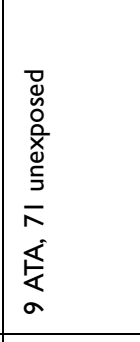 & 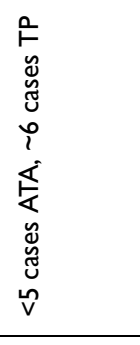 & 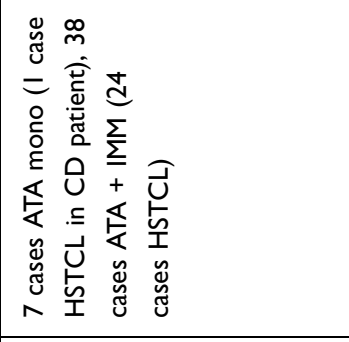 & 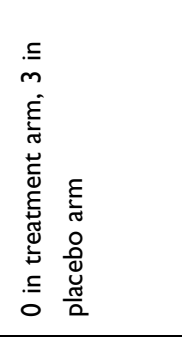 & 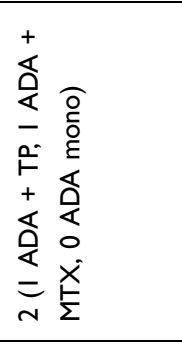 & 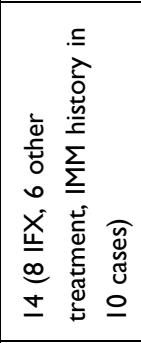 & 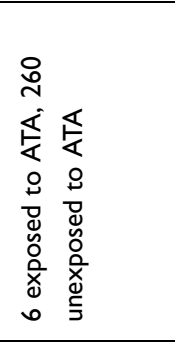 \\
\hline 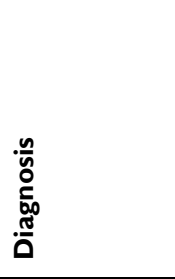 & 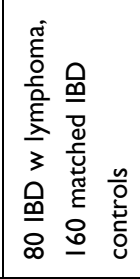 & 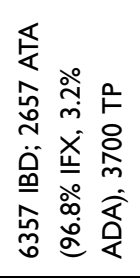 & 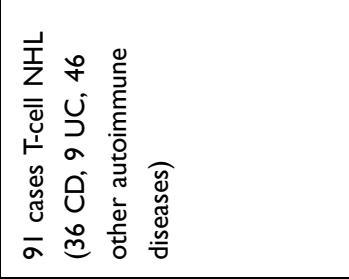 & 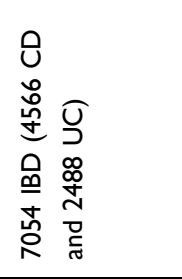 & 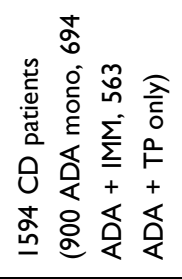 & 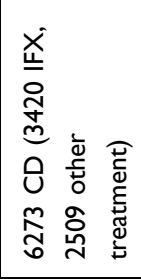 & 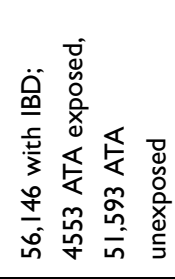 \\
\hline 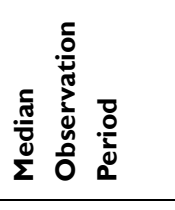 & & $\begin{array}{l}\stackrel{u}{\Sigma} \\
0 \\
o \\
\underline{0} \\
\underline{0}\end{array}$ & ' & 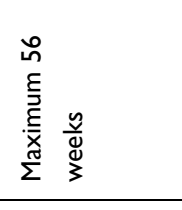 & $\frac{n}{\underline{\lambda}}$ & $\begin{array}{l}\stackrel{n}{\lambda} \\
\stackrel{n}{n}\end{array}$ & $\begin{array}{l}\stackrel{n}{\lambda} \\
\hat{m}\end{array}$ \\
\hline 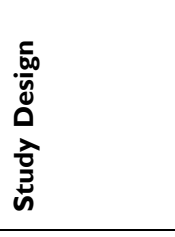 & 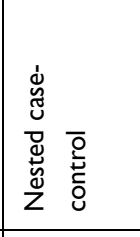 & 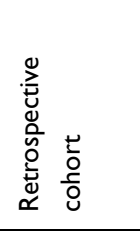 & 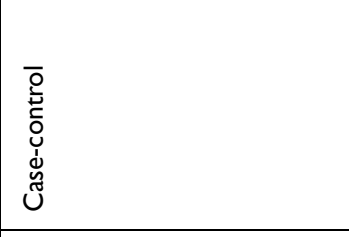 & 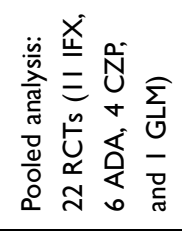 & 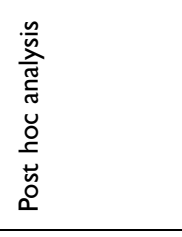 & 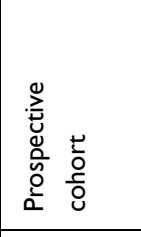 & 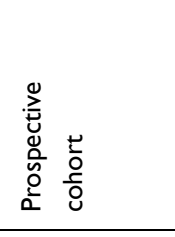 \\
\hline 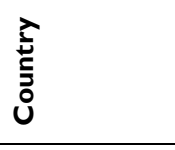 & $\check{د}$ & 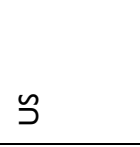 & $\breve{د}$ & 弚 & 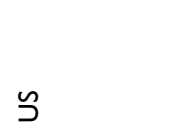 & $\check{د}$ & 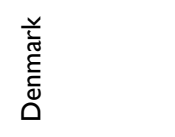 \\
\hline 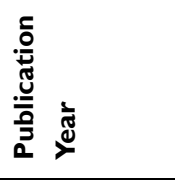 & $\frac{m}{i}$ & $\frac{m}{i}$ & $\frac{m}{i}$ & $\frac{\nabla}{i}$ & $\frac{\nabla}{i}$ & $\underset{+}{+}$ & $\frac{\nabla}{i}$ \\
\hline $\begin{array}{l}\frac{\partial}{\partial} \\
\frac{1}{y} \\
\dot{y}\end{array}$ & 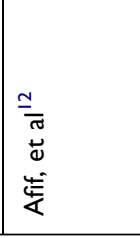 & 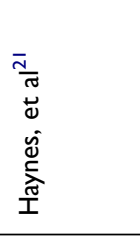 & 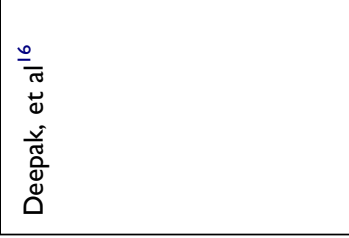 & 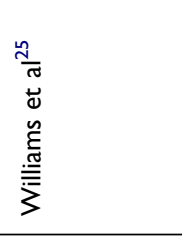 & 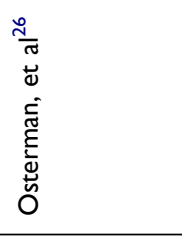 & 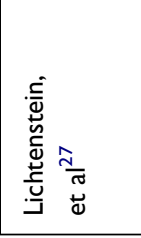 & 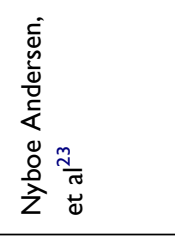 \\
\hline
\end{tabular}




\begin{tabular}{|c|c|c|c|c|c|c|}
\hline 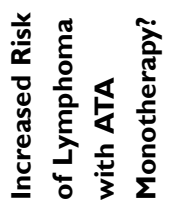 & $\stackrel{\circ}{z}$ & $\stackrel{0}{z}$ & 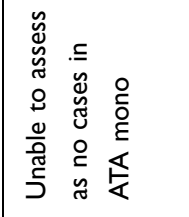 & $\stackrel{0}{z}$ & 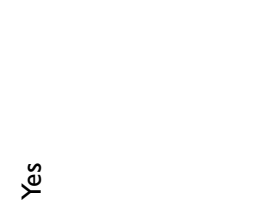 & 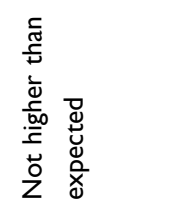 \\
\hline 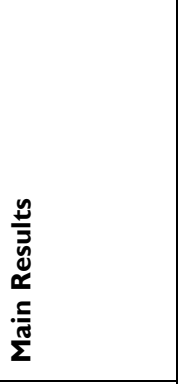 & 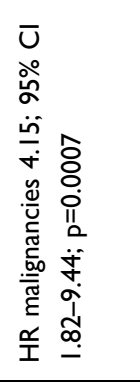 & 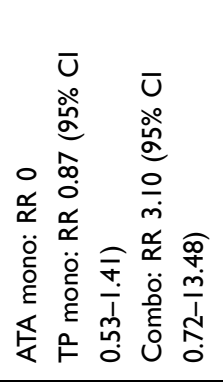 & 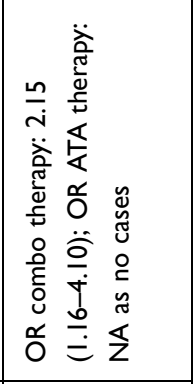 & 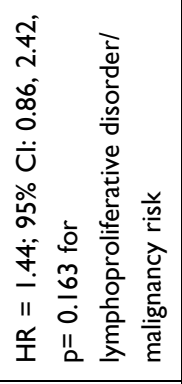 & 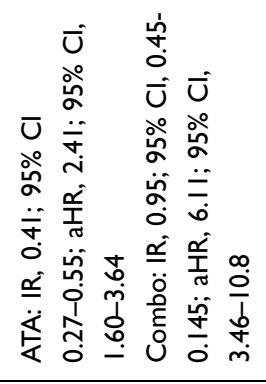 & 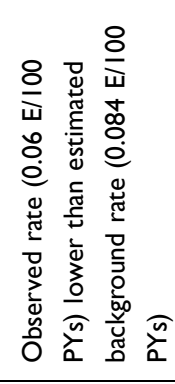 \\
\hline 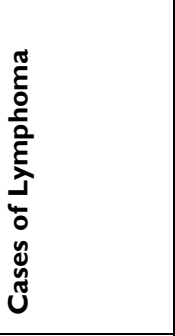 & 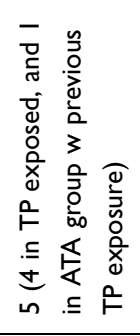 & 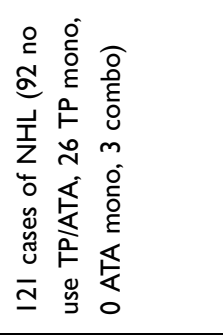 & 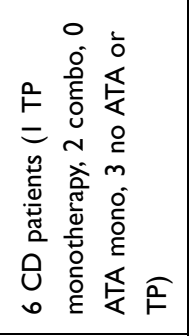 & 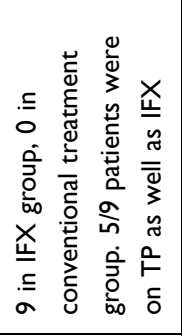 & 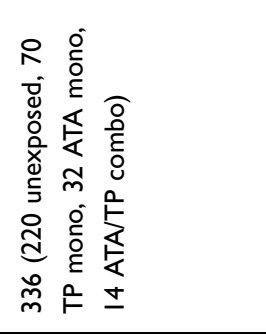 & 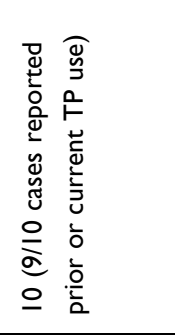 \\
\hline 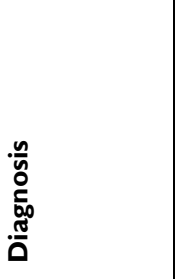 & 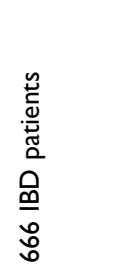 & 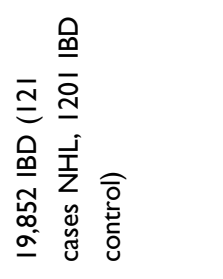 & 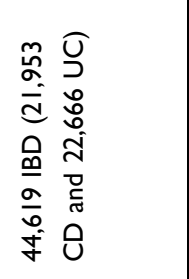 & 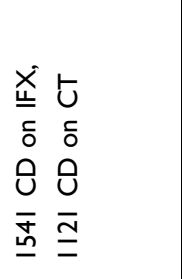 & 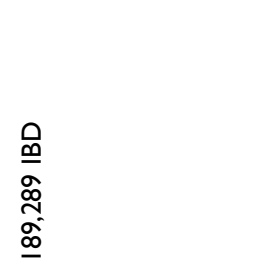 & 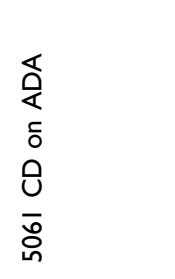 \\
\hline 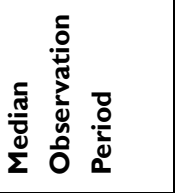 & $\underset{\nabla}{\tilde{\lambda}}$ & ' & & 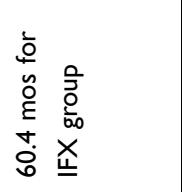 & 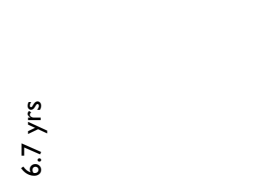 & $\begin{array}{l}\stackrel{n}{\lambda} \\
m \\
i\end{array}$ \\
\hline 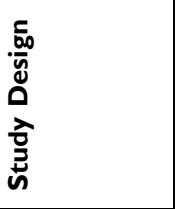 & 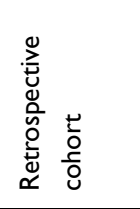 & 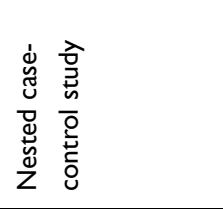 & 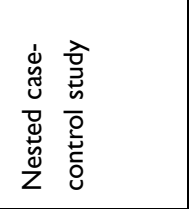 & 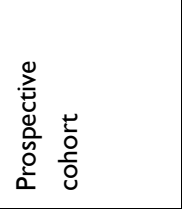 & 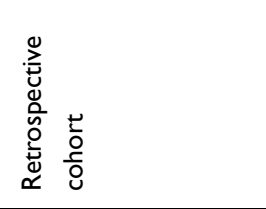 & 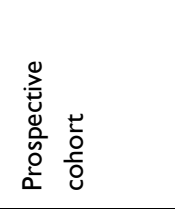 \\
\hline 站 & 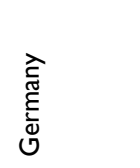 & 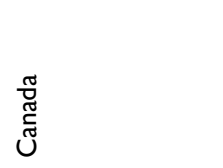 & 좊 & 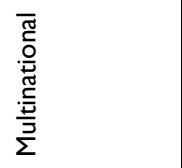 & 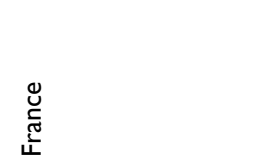 & 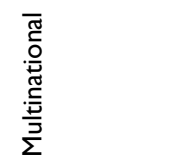 \\
\hline 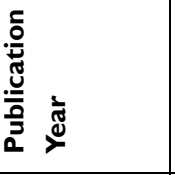 & $\stackrel{ \pm}{\circ}$ & $\frac{n}{\grave{N}}$ & $\stackrel{\circ}{\frac{N}{2}}$ & $\overline{\bar{\nu}}$ & $\overline{\bar{i}}$ & $\frac{\infty}{2}$ \\
\hline 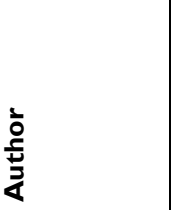 & 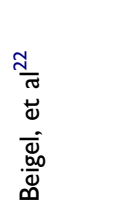 & 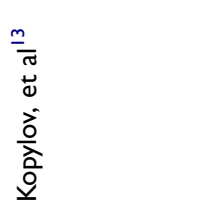 & 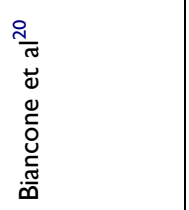 & 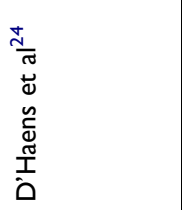 & 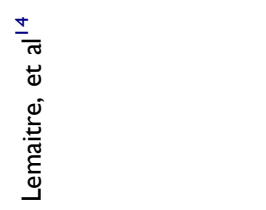 & 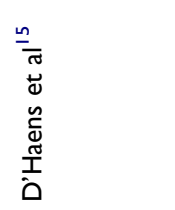 \\
\hline
\end{tabular}




\begin{tabular}{|c|c|c|}
\hline$\stackrel{\circ}{z}$ & . & ㅇ \\
\hline 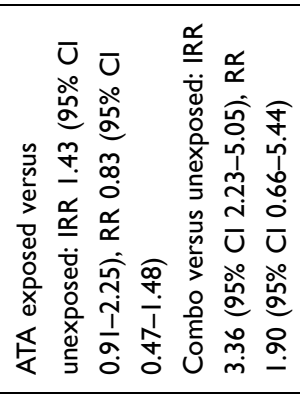 & 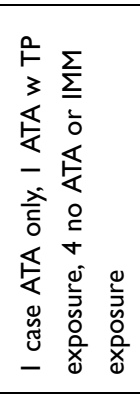 & 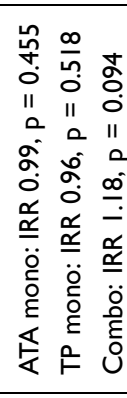 \\
\hline 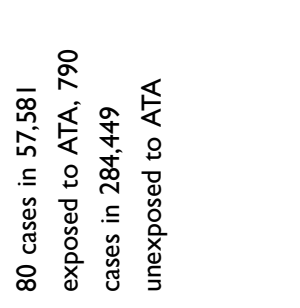 & 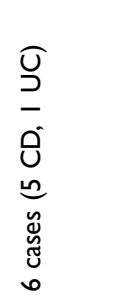 & 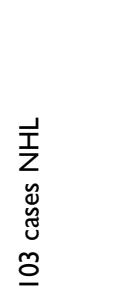 \\
\hline 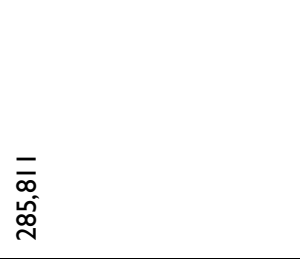 & $\frac{\mathrm{O}}{8}$ & 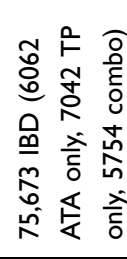 \\
\hline 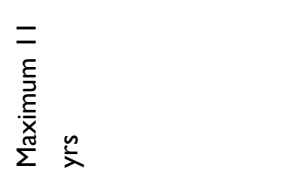 & & 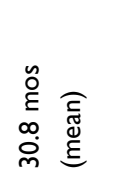 \\
\hline 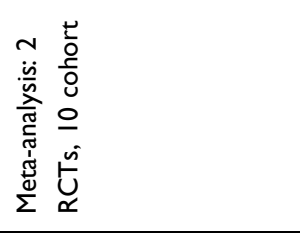 & 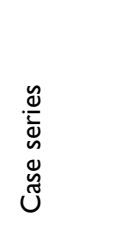 & 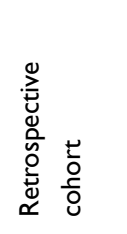 \\
\hline 昙 & $\begin{array}{l}\overline{\widetilde{a}} \\
\text { a. } \\
\overrightarrow{\vec{E}} \\
0 \\
0\end{array}$ & $\begin{array}{l}\text { 志 } \\
\text { I }\end{array}$ \\
\hline$\frac{\infty}{\grave{N}}$ & $\frac{\infty}{\grave{N}}$ & ్ㅗ \\
\hline 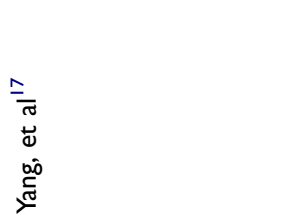 & 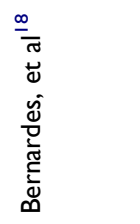 & 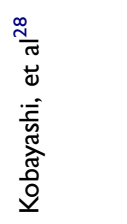 \\
\hline
\end{tabular}

such as azathioprine, 6-mercaptopurine, and methotrexate, while thiopurines (TP) included azathioprine or 6-mercaptopurine. Combination (combo) therapy alludes to ATA therapy plus IMM or TP therapy.

\section{Lymphoma as a Specific Outcome}

Seven studies assessed lymphoma as a specific outcome. ${ }^{12-18}$ Only Lemaitre et al found an associated small but increased risk in lymphoma development in ATA monotherapy. ${ }^{14}$ However, as mentioned in a similar systematic review by Ferraro et al, its analysis was limited by lag-time bias, meaning that a case of lymphoma would be considered part of the exposure group, even if lymphoma was diagnosed within a few days of starting ATA therapy. ${ }^{19}$ The authors did perform a sensitivity analysis, introducing a lag time of 3 to 6 months but, as Ferraro et al described, this could lead to further bias within the sample by decreasing the number of lymphoma cases in the unexposed group (ie, cases excluded using parameters of the sensitivity analysis were eliminated from analysis altogether and were not considered to be cases to include in the unexposed group's numbers).

The meta-analysis performed by Yang et al did not appreciate an increased relative risk of lymphoma with ATA monotherapy (random effects: $\mathrm{RR}=1.00,95 \% \mathrm{CI}$, 0.39-2.59; $\mathrm{p}=0.996$ ). However, there was an increased incidence rate ratio when comparing ATA monotherapy versus those unexposed to either IMM or ATA (IRR 1.65 [95\% CI, 1.16-2.35; $\mathrm{p}=0.006]) .{ }^{17}$ The authors point out, however, that not all studies included had a long enough duration of follow-up or large sample sizes. Some studies had as little as 1 year follow-up time. Furthermore, they noted that previous or concurrent exposure to other biologics may have been missed in the control as well as experimental groups, possibly affecting data interpretation.

Three additional studies (two nested case-control and one cohort) and an observational study did not find an associated increased risk of lymphoma with ATA monotherapy, ${ }^{12,13,15,18}$ A case-control study by Deepak et al also did not find an association between lymphoma and ATA monotherapy risk, although IBD was not the only autoimmune condition assessed in that study. ${ }^{16}$

Four studies assessed the risk of lymphoma development with combination (ATA +IMM or TP) therapy. ${ }^{13,14,16,17}$ Similar to the CESAME and Kaiser studies, three papers found a significantly increased risk associated with combination therapy or previous TP 
exposure. ${ }^{14,16,17}$ This finding was not noted in the study by Kopylov et $\mathrm{al}^{13}$ Although relative risk was elevated in the combination therapy group (RR 3.10 (95\% CI $0.72-$ 13.48)) when compared to TP (RR 0.87 (95\% CI $0.53-$ 1.41)) or ATA monotherapy (RR 0), it did not reach clinical significance (Table 1).

\section{Lymphoma as Composite Outcome}

Nine studies assed malignancy as the primary outcome. ${ }^{20-28}$ Of those, seven included lymphoma as a separate composite outcome. $^{20,21,23,25-28}$ Two of the seven studies had no cases of lymphoma appreciated in the ATA monotherapy group. ${ }^{22,24}$ Of those studies that looked at lymphoma as a composite outcome and had cases in a setting of ATA monotherapy, none appreciated an increased risk of lymphoma.

The Danish nationwide registry-based cohort study collected malignancy data on greater than 56,000 IBD patients, comparing those exposed to ATA therapy and ATA-naïve patients from 1999 to $2012 .^{23}$ Eighty-one out of 4553 ATAexposed patients $(1.8 \%)$ who developed cancer (median follow-up 3.7 years) whereas 3465 of 51,593 ATA-naïve patients (6.7\%) developed cancer (aRR 1.07, 95\% CI 0.85-1.36). Sub-analyses assessing time to cancer development since first ATA exposure ( $<1$ year, 1 to $<2$ years, 2 to $<5$ years, 5+ years) and number of ATA doses did not result in significantly increased risk of cancer. Furthermore, no specific cancers stood out as being more prevalent. Relative risks of site-specific cancers decreased when adjustments were made for azathioprine exposure, as $85 \%$ of the patients sampled had received this medication. Relative risk for lymphoma (8 cases) was 1.36 (95\% CI, 0.67-2.76), which decreased to 0.90 (95\% CI, 0.42-1.91) when adjusted for azathioprine. Many of the patients included had received other IBD medications during their study follow-up including 5-ASA $(80 \%)$ and oral corticosteroids (91\%). Furthermore, power was limited in subgroup analyses for site-specific malignancies due to too few cases.

\section{Discussion}

The results section highlighted recent articles assessing the risk of ATA monotherapy with lymphoma development, along with including the articles' additional data regarding TP monotherapy and combination therapy lymphoma risk. In addition, there is a brief assessment of some of the methodological flaws. The discussion section is devoted to summarizing ATA monotherapy lymphoma risk along with additional review topics such as baseline IBD lymphoma risk, ATA therapy in patients with current or previous cancer, and the associations of ATA monotherapy with the development of HSTCL, a rare but aggressive and almost universally fatal disease. This section will conclude with a discussion about the implications of patient care and medication selection.

\section{TNF-a Antagonist Exposure in IBD Patients and Implications for Lymphoma Risk}

Overall, there appears to be little to no increased risk of lymphoma development in the setting of ATA monotherapy. The few studies that showed an increased, but small, risk had concerning methodological limitations that may have affected conclusions.

Previously, long term, prospective data on ATA use and risk for lymphoma had been difficult to obtain due to the relative infancy of the medication and the potential confounding effects of TP use. Many of the studies mentioned in this review benefitted from data that had a follow-up period greater than five years and had a greater proportion of ATA monotherapy patients with no previous TP exposure. Additionally, attempts have been made to address this through community and academic-based registry data. One such registry is the Crohn's Therapy, Resource, Evaluation, and Assessment Tool (TREAT) registry. Initial data from 2006 showed mortality rates to be no different between IFX and non-IFX-treated patient groups after short term follow-up of 1.9 years. ${ }^{29}$ Further assessment in 2014 (follow-up time of 7.6 years) showed no therapies (IMM, IFX, or combination therapy) to be an independent risk factor for malignancy, although age, disease duration, and smoking status were. ${ }^{27}$ An exposure-based analysis did identify a numerically-increased risk associated with IMM use alone (OR 4.19) and combination therapy (OR 3.33), but not with IFX monotherapy (OR 1.96). However, this was not statistically-significant in any of the medication groups. It should be mentioned that the registry was started soon after IFX was available for IBD treatment. This is an important consideration due to the relatively small number of patients in the registry on this therapy alone without any previous exposure to TP or other immunosuppressive therapy.

This point is further emphasized by the systematic review with meta-analysis performed by Williams et al in $2014 .^{25}$ Data were pooled from all available placebocontrolled trials to evaluate the risk of malignancy with ATA therapy in IBD with subgroup analysis focusing on 
lymphoma risk. This was difficult to perform due to underpowering. Out of 22 eligible randomized control trials including 7054 patients, there were 16 malignancies $(0.39 \%)$ in 4135 IBD patients on ATA therapy versus 13 malignancies $(0.45 \%)$ in 2919 placebo patients. Therefore, relative risk of malignancy was not statistically different between the two $(R R=0.77)$. Assessment of lymphoma cases proved difficult due to previous ATA exposure in the placebo group, wide variances of previous TP exposure amongst evaluated studies (anywhere from 16\% to $100 \%$ of controls and $20-100 \%$ of ATA monotherapy patients received concomitant IMM in certain studies), and short follow-up time. Additionally, patients were considered to be exposed to a treatment if malignancy cases occurred at least 14 days after drug exposure.

Similarly, in a pooled retrospective analysis of six randomized placebo-controlled trials assessing adalimumab (ADA) efficacy in CD, Osterman et al, did not appreciate an increased risk of malignancy with ADA monotherapy compared to the general population. ${ }^{26}$ Two cases of lymphoma were reported, one in the ADA/TP combination group $(n=563)$ and one in the ADA/any immunomodulatory group $(n=694)$. No cases of lymphoma were appreciated in the ADA monotherapy group $(n=900)$ although median follow-up time of the study was short (1.5 years).

Our review provides a wide range of study designs, including meta-analyses, case-control series, retrospective and prospective cohort studies, cross-sectional studies, and a post hoc analysis. As has been mentioned, lymphoma risk in relation to ATA therapy has been a widely assessed topic, with previous studies using multiple analytic methods. Our review of studies since 2013 demonstrates this heterogeneity in study design and results.

\section{Baseline Risk of Lymphoma in IBD}

When evaluating if a therapy increases malignancy risk, it is important to acknowledge whether the underlying inflammatory disorder has its own malignancy risk and acknowledge this contribution when assessing therapies meant to control severe inflammatory disease. For example, rheumatoid arthritis appears to possess an inherently elevated risk for development of lymphoma due to the disease itself. ${ }^{6}$ In IBD, concern lies whether higher burden of disease, such as with severe inflammation in stricturing and fistulizing $\mathrm{CD}$, may be driving this increased risk, rather than the medications meant to try to control inflammation.
Many studies have sought to assess this relation with conflicting results. The European Crohns and Colitis Organization evidence-based consensus (ECCO) assessed this concern, in 2015. ${ }^{30}$ Their overall consensus was that IBD patients showed a trend toward higher risks of developing hematological malignancies, more specifically leukemia in $\mathrm{UC}$ and lymphoma in $\mathrm{CD}$ based on large population-based cohort studies. The authors noted that, although many previous studies did not show an overall increased risk of lymphoma development in IBD, many of these studies did not separately analyze UC and CD patients and instead pooled all IBD patients into one analysis. However, previous treatment exposures were not mentioned or controlled for in all of the cited studies that emphasized an increased risk of CD with lymphoma.

This difference in results based on IBD subtype versus pooled analysis can be seen in the subsequent examples. Herrinton et al assessed the inherent risk of lymphoproliferative disorder development associated with having an inflammatory disorder. ${ }^{10}$ When they compared a population of IBD patients on no TP or ATA therapy to a general, non-IBD population, the SIRR was $1.0(95 \% \mathrm{CI}$, 0.96-1.1), leading to the conclusion that IBD itself does not increase one's risk of developing a lymphoproliferative disorder.

Conversely, a Danish population-based cohort study, assessed patients with IBD from 1978 to 2002. ${ }^{31}$ One thousand five hundredfifteen patients with UC and 815 patients with $\mathrm{CD}$ were assessed. The authors found an overall increased risk for malignancy in $\mathrm{CD}$ patients never exposed to TP (SIR 1.37 95\% CI 1.07-1.73) but not in UC patients unexposed to TP (SIR 1.12 95\% CI 0.96-1.30). An increased risk of lymphoma was appreciated in patients with CD (SIR 3.01; 95\% CI 1.21-6.19), especially non-Hodgkin lymphoma, and was related to young age at $\mathrm{CD}$ diagnosis, combined small bowel and colonic disease, and lack of 5-ASA use. There was no higher risk of lymphoma appreciated in patient exposed (SIR 2.85; 85\% CI 0.35-10.30) or unexposed (SIR 3.73; 95\% CI 1.21-8.70) to TP. There was no appreciable increased risk of lymphoma with UC patients.

Similar findings were appreciated in a Dutch cohort study by van de Heuvalet al which evaluated malignancy data from 1991 to 2013 in 2801 IBD patients. ${ }^{32}$ Overall, regardless of medication exposure or class of drug, those with $\mathrm{CD}$ had a higher risk of hematologic malignancies. Those with UC, however, had no increased risk for extraintestinal malignancy. Subgroup analysis of medication 
exposure in IBD patients showed slightly different findings. The authors subdivided IBD patients based on current and previous therapies. This included any immunosuppression exposure (biologics, TP, or methotrexate), TP exposure alone, or no IS exposure (IS-naïve) - Increased risk for hematologic malignancy was appreciated in the IS (SIR 3.12, CI 1.14-6.79) and the TP alone (SIR 4.94, CI 1.80-10.74) groups. However, in IS-naïve patients, there was not an appreciably increased risk (SIR 1.00, CI 0.48-1.84).

\section{Role of EBV in Lymphoma}

The role of EBV predisposing IBD patients to malignancy in the setting of immunosuppressant use has been a topic of debate. The concern lies in the virus promoting tumor development because the immune system is inactive as a result of immunosuppressive therapy.

While the role of EBV in the development of several B-cell lymphomas, such as Burkitt's lymphoma, AIDS lymphomas, nasopharyngeal carcinoma, gastric carcinoma, and post-transplant lymphoproliferative disorder, is well known, it is still unclear if and how EBV contributes to lymphoma risk in IBD patients on immunosuppression. This is in part due to limited data as those with malignancy have often been excluded from studies.

In a retrospective study by Reeson et al from 2005 to 2015, 14 out of 209 participants enrolled developed lymphoproliferative disorders. $^{33}$ Those studied were on biologics, IMM, or combination therapy. All patients with lymphoma were male and, interestingly, only three had a positive serology for EBV. This study is continuing to collect prospective data to delineate the association of IS use in IBD and role of EBV in lymphoma development.

A subsequent review article in 2019 by Shivaji et al cited observational studies attributing TP and ATA use as risk factors for the development of EBV-associated lymphoproliferative disorders. ${ }^{34}$ It must be noted, however, that the risk was primarily attributed to TP use and overall risk was small. In this review, it was recommended that EBV serology be considered before starting these agents given that no EBV vaccine is available and prophylaxis is not suggested with the low overall risk of lymphoma. However, they did mention serologies may be helpful in guiding therapy decisions while considering other baseline patient characteristics that may increase lymphoma development risk. ${ }^{35}$ If a patient were to develop EBV while on these agents, discontinuation of therapy was encouraged. At present, given the unclear nature of this risk due to limited data, screening for EBV is not yet standard of care in managing those with IBD. ${ }^{34}$

Further support to continue ATA management was reported in a 2014 review. $^{36}$ It recommended continuing ATA use in the setting of acute EBV infection or reactivation unless severe disease sequelae or significant viremia occurred. Severe sequelae included lymphoma, mononucleosis, hepatitis, bone marrow suppression, and severe forms of hemophagocytic lymphohistiocytosis.

\section{Hepatosplenic T-Cell Lymphoma and Anti-TNF Therapy}

A more well-supported association between IMM use in IBD and malignancy development is that of hepatosplenic T-cell lymphoma (HSTCL), a rare malignancy with rapid progression and high mortality rates. HSTCL has been associated with IFX, ADA, and, primarily, TP use. $^{34}$ In 2011, Kotylar et al used the FDA Adverse Event Reporting System (AERS) database to assess the risk of HSTCL with ATA use. ${ }^{37}$ Of the 36 subjects diagnosed with HSTCL, 16 were on TP monotherapy and 20 had both TP and ATA exposure. Median time from start of TP therapy to diagnosis of HSTCL was not statistically different between combination therapy and TP monotherapy at 5.5 and 6 years, respectively. Further review in 2019 found that the risk for HSTCL was indeed related primarily to TP use. ${ }^{38}$ The risk has also been attributed to dual therapy, especially if used for longer than 2 years. $^{36}$

A 2020 study also examined data from the FDA AERS database. ${ }^{39}$ Patients were divided into combination therapy, biologic monotherapy, or no biologic therapy groups. Sixty-two cases of HSTCL were identified from 2002 to 2017 with the majority of patients being male (83.6\%), young (median age 28 (range 12-81)), and having Crohn's disease $(84.7 \%)$. All affected patients had received at least one dose of a biologic (ATA, IL 12/23, or integrin inhibitor). Fifty-seven patients had azathioprine or mercaptopurine exposure. The five patients who had no history of TP use had IFX exposure. The authors argued that, despite the appreciated correlation with TP use, there still may be an associated risk with ATA. ${ }^{39}$

The study also assessed the role of EBV in HSTCL development. Only 4 of the 62 cases were found to have active EBV infection. Therefore, the role of EBV in HSTCL remains unclear. While HSTCL is a serious illness with poor outcomes, the rarity of developing this 
malignancy should be weighed against the higher risk of worsening or uncontrolled IBD when considering ATA therapy. ${ }^{39}$

\section{Prior or Current History of Malignancy and Management Strategies}

Another area of clinical concern involves IBD medication management in a patient with prior history of or current malignancy. Data on this topic have been limited as most randomized control trials containing IBD patients on ATA therapy exclude those with a prior history of cancer. ${ }^{40}$ A systematic review and meta-analysis of 16 studies from 2016 assessed rates of recurrent or new malignancy in 11,702 individuals with IBD, RA, and psoriasis. Ten of these sixteen studies included control groups of patients not exposed to immunosuppression. There was no significant difference between pooled incidence rates in ATA (33.8/1000 person years), IMM (36.2/ 1000 person years), or no immunosuppressive therapy $(37.5 / 1000$ person years) in cancer development ( $p>0.1$ for all). There was an increased, although non-significant, rate of recurrent malignancy in those on combination immune suppression $(54.5 / 1000$ person years; $\mathrm{p}>0.1)$. There were decreased rates of new or recurrent malignancy in the ATA group compared to the IMM group in a random effects meta-analysis (incidence difference= -9.8 per 1000 person years, $95 \% \mathrm{CI}=-19.5$ to -0.1 ; $\left.\mathrm{I}^{2}=41.8 \% ; \mathrm{p}=0.089\right)$. No difference in malignancy incidence was observed in random effects model comparisons between IMM versus no immunosuppression as well as in the ATA versus no immunosuppression comparison. However, numbers of studies were low and thus statistical power was low. ${ }^{41}$

When isolating IBD and RA patients, there were similar absolute and relative risks amongst the therapy or no therapy subgroups (for IBD - no IS 35.7/1000 person years, IMM 37.9/1000 person years, ATA 48.5/1000 person years; $p>0.30)$. Given significant heterogeneity, those with skin cancer as the index malignancy were eliminated from further data analysis. Despite this, there were still similar rates of new or recurrent malignancy between all three groups.

Based on this data, there appears to be no increased risk of recurrent malignancy with ATA or IMM therapy. However one must acknowledge that propensity bias may exist as those with higher recurrence-risk malignancies may not have been restarted on immunosuppression and may not have been included in the sample. This concern was called into question in the "Letters to the Editor" from Gastroenterology, 2017. ${ }^{11}$

Several studies have also concluded that there is no increased risk of additional malignancy development (including lymphoma as a specific parameter) when using ATA therapy several years after malignancy diagnosis and treatment. ${ }^{34,41,42}$ When comparing data on cancer-free survival at 5 years amongst those on IMM monotherapy, combination therapy, ATA, or no immunosuppression, there were no differences observed. ${ }^{42}$

The optimal time to restart or initiate immunosuppressive therapy after malignancy diagnosis is varied and debatable. Current expert opinion recommends a 5-year immunosuppression holiday after index cancer diagnosis. In the work by Shelton et al, the median interval between starting immunosuppression and the index cancer was 6 years. However, in a sub-analysis of studies with intervals shorter than 6 years, there was no difference in risk of new or recurrent malignancy. ${ }^{41}$ The Axelrad study gathered data demonstrating a median time of 14.5 months between cancer diagnosis and start of ATA therapy (range 0-704 months). ${ }^{42}$ Several patients also continued ATA therapy despite cancer diagnosis. As cancer recurrence outcomes on immunosuppression did not differ from inherent baseline malignancy specific recurrence rates, this latter study may show that earlier initiation of IBD therapy may be safe. This was discussed by Sebastian et al in an article from 2019 reporting a 2-year wait period for lower-risk malignancies (which includes lymphoma) and favoring a 5-year wait for cancers with an intermediate to higher risk of recurrence. ${ }^{40}$

The European Crohn's and Colitis Organization (ECCO) guidelines support these recommendations. ${ }^{30}$ They cite data from organ transplant recipients suggesting a five-year wait period before starting immunosuppressants, especially in malignancies with intermediate to high risk of recurrence (ie, uterine, colon, prostate, breast, bladder, sarcoma, melanoma and non-melanoma skin cancer, myeloma, and symptomatic renal carcinoma). In studies of transplant recipients maintained on immunosuppression for graft maintenance, most cancer recurrences occurred within 2 years of index malignancy, while only $13 \%$ occurred after 5 years. ${ }^{40}$

As has been explored in this review, the risk of lymphoma associated with ATA use is still in question. Furthermore, as discussed above, risk of recurrent malignancy must also be considered in those with a prior history of lymphoma. Current recommendations seem to agree 
upon a few approaches to the management of these patients. First, controlling IBD is priority and the benefits of therapy may outweigh the risks of lymphoma given that absolute risk of malignancy remains low. When possible, combination therapy of IMM and ATA should be limited to no more than 2 years. ${ }^{30}$ Monotherapy with ATA, integrin inhibitors, IL $12 / 23$ agents are generally accepted for IBD management in patients with a prior history of malignancy after a risk/benefit discussion has occurred with the patient. ATA, however, should be avoided in those with active malignancy, especially those with prior history of or current melanoma, HSTCL, or with lymphoma after an acute EBV infection. ${ }^{34,36,41}$ One may consider continuing ATA in those with solid tumors and curative resection. ${ }^{36}$

\section{Implications for Therapy}

In this review, we discuss the recent salient literature from 2013 to date and describe limitations that affect current data regarding lymphoma risk in ATA monotherapy. Despite the minimal risk noted in this review, risk/benefit discussions about any therapy are a necessary component of patient care. Although a malignancy risk may be present and is a significant facet of care to acknowledge, active and poorly-controlled disease can have many negative outcomes including, but not limited to, strictures, abscesses, worsening of extraintestinal manifestations that mirror disease, malignancy, surgery, and even death.

A 2006 decision analytic model conceived and interpreted by Seigel et al assessed several outcomes in 35-year -old patients with moderate to severe active CD in two arms of 100,000 patients each: those receiving IFX and those on standard therapy (azathioprine, 6-mercaptopurine, corticosteroids). ${ }^{43}$ At the end of 1 simulated year, treatment with IFX led to 12,216 more patients in remission, 4255 fewer surgeries, and 33 fewer deaths from flares. Furthermore, quality adjusted life years (QALYs) were higher per patient in the IFX group than in the standard therapy group (0.77 QALYs/patient vs $0.75 /$ patient).

A 2009 article also assessed the rate of NHL with ATA monotherapy compared to the expected rate via the Surveillance Epidemiology and End results (SEER) database. $^{44}$ The expected rate of NHL in the SEER database was 1.9 per 10,000 patient years. Comparatively, ATA-treated subjects had an elevated risk (SIR, 3.23; 95\% CI, 1.5-6.9). Despite the significantly elevated risk, the authors emphasized that the absolute occurrence rate was low and should be weighed against the substantial benefits of treatment.

\section{Conclusions}

In sum, the risk of ATA monotherapy associated lymphoma in IBD patients has been frequently assessed. Most recent data does not appreciate an increased risk with ATA monotherapy. Rather combination therapy or previous TP exposure portends an increased risk. Some studies have appreciated a small relative risk although concerns related to methodological flaws and short follow-up time may skew results.

Future studies should be directed to addressing these limitations. When determining optimal therapy, patient and disease characteristics, as well as extent and severity of inflammation should be weighed against these potential risks when selecting therapy. This is the essence of shared decision making with patients and families.

\section{Key Points}

1. While there is a slightly elevated risk of lymphoma development with thiopurine use and anti-TNF/thiopurine combination, there is little convincing evidence to date associating anti-TNF monotherapy and lymphoma development in IBD patients.

2. Further prospective, larger sample studies are needed to address the risk of lymphoma attributed to anti-TNF monotherapy in patients unexposed to thiopurines.

3. Anti-TNFs should be avoided in the setting of prior or current melanoma, HSTCL, lymphoma after an acute EBV infection, and active malignancy. AntiTNFs may be considered for use in those with a prior history of malignancy (save for melanoma) or current solid tumor that undergoes curative resection.

4. Given the current available data and knowledge of relative versus absolute risks, risk and benefit discussions should be undertaken with patients when determining optimal IBD therapy.

5. Reviewing our current knowledge and expert opinion to date, absolute risk of lymphoma with anti-TNF use is low and benefits of adequate IBD control outweigh the potential risk of malignancy development.

\section{Funding}

There was no source of funding for this review.

\section{Disclosure}

Dr. Clarke has reviewed research grants and is a consultant for Pfizer; He is on the speaker's bureau for Pfizer, 
Janssen, Takeda and ABBvie and reports no other potential conflicts of interest for this work. Dr. Dahmus and Dr. Rosario report no conflicts of interest for this work.

\section{References}

1. Nocturne G, Boudaoud S, Ly B, Pascaud J, Paoletti A, Mariette X. Impact of anti-TNF therapy on NK cells function and on immunosurveillance against B-cell lymphomas. J Autoimmun. 2017;80: 56-64. doi:10.1016/j.jaut.2017.02.001

2. Horiuchi T, Mitoma H, Harashima S, Tsukamoto H, Shimoda T. Transmembrane TNF-alpha: structure, function and interaction with anti-TNF agents. Rheumatology. 2010;49(7):1215-1228. doi:10.10 93/rheumatology/keq031

3. Catrina AI, Trollmo C, Klint E, et al. Evidence that anti-tumor necrosis factor therapy with both etanercept and infliximab induces apoptosis in macrophages, but not lymphocytes, in rheumatoid arthritis joints: extended report. Arthritis Rheum. 2005;52(1):61-72. doi:10.1002/art.20764

4. Nesbitt A, Fossati G, Bergin M, et al. Mechanism of action of certolizumab pegol (CDP870): in vitro comparison with other antitumor necrosis factor $\alpha$ agents. Inflamm Bowel Dis. 2007;13 (11):1323-1332. doi:10.1002/ibd.20225

5. Brown SL, Greene MH, Gershon SK, Edwards ET, Braun MM. Tumor necrosis factor antagonist therapy and lymphoma development: twenty-six cases reported to the Food and Drug Administration. Arthritis Rheum. 2002;46(12):3151-3158. doi:10.1002/art.10679

6. Simon TA, Thompson A, Gandhi KK, Hochberg MC, Suissa S. Incidence of malignancy in adult patients with rheumatoid arthritis: a meta-analysis. Arthritis Res Ther. 2015;17(1):212. doi:10.1186/s13075-015-0728-9

7. Zintzaras E. The risk of lymphoma development in autoimmune diseases: a meta-analysis. Arch Intern Med. 2005;165(20):233 7-2344. doi:10.1001/archinte.165.20.2337

8. Dassopoulos T, Sultan S, Falck-Ytter YT, Inadomi JM, Hanauer SB. American Gastroenterological Association Institute Technical Review on the Use of Thiopurines, Methotrexate, and Anti-TNF- $\alpha$ Biologic Drugs for the Induction and Maintenance of Remission in Inflammatory Crohn's Disease. Gastroenterology. 2013;145(6): 1464-1465. doi:10.1053/j.gastro.2013.10.046

9. Lichtenstein GR, Feagan BG, Cohen RD, et al. Serious Infection and Mortality in Patients With Crohn's Disease: more Than 5 Years of Follow-Up in the TREAT ${ }^{\mathrm{TM}}$ Registry. Am J Gastroenterol. 2012;107 (9):1409-1422. doi:10.1038/ajg.2012.218

10. Herrinton LJ, Liu L, Weng X, Lewis JD, Hutfless S, Allison JE. Role of thiopurine and anti-TNF therapy in lymphoma in inflammatory bowel disease. Am J Gastroenterol. 2011;106(12):2146-2153. doi:10.1038/ajg.2011.283

11. Beaugerie L, Brousse N, Bouvier AM, et al. Lymphoproliferative disorders in patients receiving thiopurines for inflammatory bowel disease: a prospective observational cohort study. Lancet. 2009;374 (9701):1617-1625. doi:10.1016/S0140-6736(09)61302-7

12. Afif W, Sandborn WJ, Faubion WA, et al. Risk factors for lymphoma in patients with inflammatory bowel disease: a case-control study. Inflamm Bowel Dis. 2013;19(7):1384-1389. doi:10.1097/MIB.0b0 $13 \mathrm{e} 318281325 \mathrm{e}$

13. Kopylov U, Vutcovici M, Kezouh A, Seidman E, Bitton A, Afif W. Risk of Lymphoma, Colorectal and Skin Cancer in Patients with IBD Treated with Immunomodulators and Biologics: A Quebec Claims Database Study. Inflamm Bowel Dis. 2015;21(8):1847-1853. doi:10.1097/MIB.0000000000000457

14. Lemaitre M, Kirchgesner J, Rudnichi A, et al. Association Between Use of Thiopurines or Tumor Necrosis Factor Antagonists Alone or in Combination and Risk of Lymphoma in Patients With Inflammatory Bowel Disease. JAMA. 2017;318(17):1679-1686. doi:10.1001/jama.2017.16071
15. D'Haens G, Reinisch W, Panaccione R, et al. Lymphoma Risk and Overall Safety Profile of Adalimumab in Patients With Crohn's Disease With up to 6 Years of Follow-Up in the Pyramid Registry. Am J Gastroenterol. 2018;113(6):872-882. doi:10.1038/s41395-0180098-4

16. Deepak P, Sifuentes H, Sherid M, Stobaugh D, Sadozai Y, Ehrenpreis ED. T-cell non-Hodgkin's lymphomas reported to the FDA AERS with tumor necrosis factor-alpha (TNF- $\alpha$ ) inhibitors: results of the REFURBISH study. Am J Gastroenterol. 2013;108 (1):99-105. doi:10.1038/ajg.2012.334

17. Yang C, Huang J, Huang X, et al. Risk of Lymphoma in Patients With Inflammatory Bowel Disease Treated With Anti-tumour Necrosis Factor Alpha Agents: A Systematic Review and Meta-analysis. J Crohns Colitis. 2018;12(9):1042-1052. doi:10.1093/ecco-jcc/jjy065

18. Bernardes C, Russo P, Carvalho D, Saiote J, Ramos J. Lymphoproliferative Disorders in Inflammatory Bowel Disease Patients: is It the Drugs or the Disease. GE Port J Gastroenterol. 2018;25(4):175-178. doi:10.1159/000484440

19. Ferraro S, Leonardi L, Convertino I, Blandizzi C, Tuccori M. Is There a Risk of Lymphoma Associated With Anti-tumor Necrosis Factor Drugs in Patients With Inflammatory Bowel Disease? A Systematic Review of Observational Studies. Front Pharmacol. 2019;10:247. doi:10.3389/fphar.2019.00247

20. Biancone L, Armuzzi A, Scribano ML, et al. Inflammatory Bowel Disease Phenotype as Risk Factor for Cancer in a Prospective Multicentre Nested Case-Control IG-IBD Study. J Crohns Colitis. 2016;10(8):913-924. doi:10.1093/ecco-jcc/jjw048

21. Haynes K, Beukelman T, Curtis JR, et al. Tumor necrosis factor $\alpha$ inhibitor therapy and cancer risk in chronic immune-mediated diseases. Arthritis Rheum. 2013;65(1):48-58. doi:10.1002/art.37740

22. Beigel F, Steinborn A, Schnitzler F, et al. Risk of malignancies in patients with inflammatory bowel disease treated with thiopurines or anti-TNF alpha antibodies. Pharmacoepidemiol Drug Saf. 2014;23 (7):735-744. doi:10.1002/pds.3621

23. Nyboe Andersen N, Pasternak B, Basit S, et al. Association between tumor necrosis factor- $\alpha$ antagonists and risk of cancer in patients with inflammatory bowel disease. JAMA. 2014;311(23):2406-2413. doi:10.1001/jama.2014.5613

24. D'Haens G, Reinisch W, Colombel JF, et al. Five-year Safety Data From ENCORE, a European Observational Safety Registry for Adults With Crohn's Disease Treated With Infliximab [Remicade ${ }^{\circledR}$ ] or Conventional Therapy. J Crohns Colitis. 2017;11(6):680-689. doi:10.1093/ecco-jcc/jjw221

25. Williams CJ, Peyrin-Biroulet L, Ford AC. Systematic review with meta-analysis: malignancies with anti-tumour necrosis factor- $\alpha$ therapy in inflammatory bowel disease. Aliment Pharmacol Ther. 2014;39(5):447-458. doi:10.1111/apt.12624

26. Osterman MT, Sandborn WJ, Colombel JF, et al. Increased risk of malignancy with adalimumab combination therapy, compared with monotherapy, for Crohn's disease. Gastroenterology. 2014;146 (4):941-949. doi:10.1053/j.gastro.2013.12.025

27. Lichtenstein GR, Feagan BG, Cohen RD, et al. Drug Therapies and the Risk of Malignancy in Crohn's Disease: results From the TREATTM Registry. Am J Gastroenterol. 2014;109(2):212-223. doi:10.1038/ajg.2013.441

28. Kobayashi T, Uda A, Udagawa E, Hibi T. Lack of Increased Risk of Lymphoma by Thiopurines or Biologics in Japanese Patients with Inflammatory Bowel Disease: A Large-Scale Administrative Database Analysis. J Crohns Colitis. 2019. doi:10.1093/ecco-jcc/jjz204

29. Lichtenstein GR, Feagan BG, Cohen RD, et al. Serious infections and mortality in association with therapies for Crohn's disease: TREAT registry. Clin Gastroenterol Hepatol. 2006;4(5):621-630. doi:10.10 16/j.cgh.2006.03.002

30. Annese V, Beaugerie L, Egan L, et al. European Evidence-based Consensus: inflammatory Bowel Disease and Malignancies. J Crohns Colitis. 2015;9(11):945-965. doi:10.1093/ecco-jcc/jjv141 
31. Jess T, Horváth-Puhó E, Fallingborg J, Rasmussen HH, Jacobsen BA. Cancer risk in inflammatory bowel disease according to patient phenotype and treatment: a Danish population-based cohort study. Am J Gastroenterol. 2013;108(12):1869-1876. doi:10.1038/ajg.20 13.249

32. van den Heuvel TRA, Wintjens DSJ, Jeuring SFG, et al. Inflammatory bowel disease, cancer and medication: cancer risk in the Dutch population-based IBDSL cohort. Int J Cancer. 2016;139 (6):1270-1280. doi:10.1002/ijc.30183

33. Reeson M, Ambrosio L, Lam G, et al. A148 EBV STATUS AND IMMUNOSUPPRESSANT USE IN IBD PATIENTS WHO SUBSEQUENTLY DEVELOP LYMPHOMA: A RETROSPECTIVE AND PROSPECTIVE STUDY. Journal of the Canadian Association of Gastroenterology. 2018;1(suppl_2):221-222. doi:10.1093/jcag/gwy0 09.148

34. Shivaji UN, Sharratt CL, Thomas T, et al. Review article: managing the adverse events caused by anti-TNF therapy in inflammatory bowel disease. Aliment Pharmacol Ther. 2019;49(6):664-680. doi:10.1111/apt.15097

35. Rahier JF, Magro F, Abreu C, et al. Second European evidence-based consensus on the prevention, diagnosis and management of opportunistic infections in inflammatory bowel disease. J Crohns Colitis. 2014;8(6):443-468. doi:10.1016/j.crohns.2013.12.013

36. Swoger JM, Regueiro M. Stopping, continuing, or restarting immunomodulators and biologics when an infection or malignancy develops. Inflamm Bowel Dis. 2014;20(5):926-935. doi:10.1097/ MIB.0000000000000002

37. Kotlyar DS, Osterman MT, Diamond RH, et al. A systematic review of factors that contribute to hepatosplenic T-cell lymphoma in patients with inflammatory bowel disease. Clin Gastroenterol Hepatol. 2011;9(1):36-41.e1. doi:10.1016/j.cgh.2010.09.016
38. Click B, Regueiro M. Managing Risks with Biologics. Curr Gastroenterol Rep. 2019;21(1):1. doi:10.1007/s11894-019-0669-6

39. Shah ED, Coburn ES, Nayyar A, Lee KJ, Koliani-Pace JL, Siegel CA. Systematic review: hepatosplenic T-cell lymphoma on biologic therapy for inflammatory bowel disease, including data from the Food and Drug Administration Adverse Event Reporting System. Aliment Pharmacol Ther. 2020;51(5):527-533. doi:10.1111/apt.15637

40. Sebastian S, Neilaj S. Practical guidance for the management of inflammatory bowel disease in patients with cancer. Therap Adv Gastroenterol. 2019;12:1756284818817293. doi:10.1177/175628481 8817293

41. Shelton E, Laharie D, Scott FI, et al. Cancer Recurrence Following Immune-Suppressive Therapies in Patients With Immune-Mediated Diseases: A Systematic Review and Meta-analysis. Gastroenterology. 2016;151(1):97-109.e4. doi:10.1053/j.gastro.2016.03.037

42. Axelrad J, Bernheim O, Colombel J-F, et al. Risk of New or Recurrent Cancer in Patients With Inflammatory Bowel Disease and Previous Cancer Exposed to Immunosuppressive and Anti-Tumor Necrosis Factor Agents. Clin Gastroenterol Hepatol. 2016;14 (1):58-64. doi:10.1016/j.cgh.2015.07.037

43. Siegel CA, Hur C, Korzenik JR, Gazelle GS, Sands BE. Risks and benefits of infliximab for the treatment of Crohn's disease. Clin Gastroenterol Hepatol. 2006;4(8):1017-1024. doi:10.1016/j.cgh.20 06.05 .020

44. Siegel CA, Marden SM, Persing SM, Larson RJ, Sands BE. Risk of Lymphoma Associated With Combination Anti-Tumor Necrosis Factor and Immunomodulator Therapy for the Treatment of Crohn's Disease: A Meta-Analysis. Clin Gastroenterol Hepatol. 2009;7 (8):874-881. doi:10.1016/j.cgh.2009.01.004
Clinical and Experimental Gastroenterology

\section{Publish your work in this journal}

Clinical and Experimental Gastroenterology is an international, peerreviewed, open access, online journal publishing original research, reports, editorials, reviews and commentaries on all aspects of gastroenterology in the clinic and laboratory. This journal is indexed on American Chemical Society's Chemical Abstracts Service (CAS).
The manuscript management system is completely online and includes a very quick and fair peer-review system, which is all easy to use. Visit http://www.dovepress.com/testimonials.php to read real quotes from published authors. 